\title{
Die EU braucht eine klare USA-China-Strategie
}

Die Dynamik in den Wirtschaftsbeziehungen zwischen den drei großen Wirtschaftsmächten EU, USA, China hat sich in den vergangenen Monaten rasant verändert. Noch Ende 2020 sah die Welt anders aus. Die Europäische Union hatte zu diesem Zeitpunkt die Verhandlungen für ein umfassendes Investitionsabkommen mit China erfolgreich abgeschlossen. Das von den USA kritisierte Investitionsabkommen sollte die Zusammenarbeit im Handel und bei Investitionen zwischen China und der EU stärken. Und nach turbulenten Jahren unter dem ehemaligen US-Präsidenten Donald Trump gab die Wahl von Joe Biden Anlass zur Hoffnung für die Zukunft der multilateralen Beziehungen.

Nur ein paar Monate später hat sich der Wind deutlich gedreht. Die neue US-Regierung hat den harten Ton der Trump-Administration gegenüber China nicht geändert, was allerdings nicht völlig überraschen kann (Flach, 2020). Auch zwischen China und Europa haben die Beziehungen nach dem Eskalationsschritt durch Strafsanktionen Chinas einen deutlichen Dämpfer erhalten. Die chinesischen Sanktionen gegen europäische Institutionen und EU-Abgeordnete wurden als Antwort auf die personenbezogenen Sanktionen der EU gegen Verantwortliche für die Unterdrückung der Uiguren verhängt. Als eine direkte Folge dieser Eskalation wurde die Ratifizierung des chinesisch-europäischen Investitionsabkommens vorerst auf Eis gelegt. In der Zwischenzeit hat China seinen Fünfjahresplan vorgestellt, der einen starken Fokus auf die Entwicklung eigener technologischer Fähigkeiten und auf eine Reduzierung seiner Abhängigkeit von anderen Staaten und Unternehmen legt. Importe aus dem Westen sollen zunehmend verringert und durch im Inland produzierte Waren ersetzt werden. Diese neue Entwicklung hat die EU wieder näher an die USA herangeführt. Bedeutet die Annäherung an die USA eine grundsätzliche Änderung der europäischen Strategie gegenüber der wachsenden Macht Chinas? Was wären die Folgen einer weiteren Eskalation mit China? Was bedeutet die US-amerikanische Strategie gegenüber China für die EU?

\section{Die neue transatlantische Agenda wird mehr von Europa verlangen}

Die Tatsache, dass es im internationalen Handel keine Rückkehr zu business as usual geben wird, hat Auswirkungen auf die Wirtschaftsbeziehungen zwischen Europa und China. Die USA werden ein schärferes Vorgehen von Europa gegen Chinas Handelspraktiken fordern: Die Jahre 2016 bis 2020 unter dem ehemaligen US-Präsidenten Trump haben Europa wenige Zugeständnisse in Bezug auf Chinas Praktiken und Strategie abverlangt, denn es war ohnehin klar, dass unter Trump kein multilaterales Vorgehen möglich sein würde. Das ist jetzt anders: Die USA erwarten nun unter USPräsident Biden eine klare Positionierung der EU gegenüber China.

US-Präsident Biden hat während seines Wahlkampfs verkündet, dass er eine stärkere wirtschaftliche Integration mit Europa vorantreiben will. Ein zentrales Element der Zusammenarbeit zwischen den USA und der EU sollte sein, gemeinsam eine WTO-Reform anzuleiten. In der im Februar veröffentlichten EU-Handelsstrategie wird betont, dass die Zusammenarbeit mit den USA entscheidend sein wird, um sicherzustellen, dass das internationale Regelwerk „im Einklang mit den Werten einer demokratischen, offenen und integrativen Gesellschaft" steht (European Commission, 2021). Vorstellbar ist auch eine Zusammenarbeit in Bereichen wie Umwelt, Menschenrechte oder geopolitische Fragen. Doch mittlerweile muss den Europäer:innen klar sein,

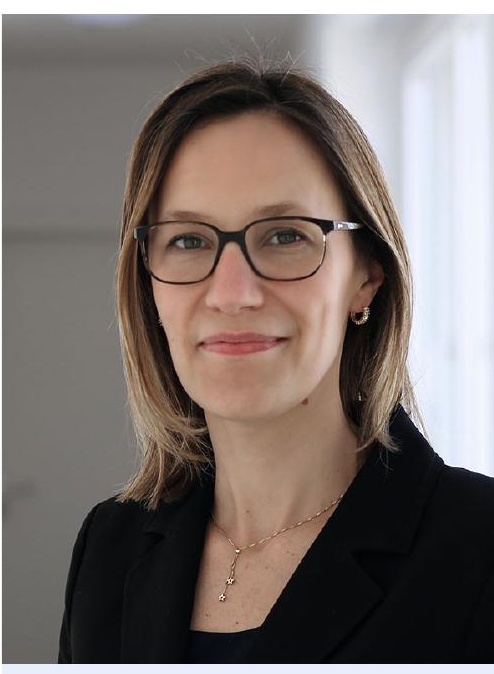

Lisandra Flach ist Leiterin des ifo Zentrums für Außenwirtschaft und Professorin für Volkswirtschaftslehre, insbesondere Ökonomik der Globalisierung, an der Ludwig-Maximilians-Universität München. 
dass es angesichts Bidens protektionistischer Agenda aktuell wenig Hoffnung auf ein Handelsabkommen mit den USA gibt. So betont das Parteiprogramm der Demokraten, dass die USA „keine neuen Handelsabkommen aushandeln werden, bevor sie nicht zuerst in die amerikanische Wettbewerbsfähigkeit im eigenen Land investiert haben“ (Democratic Party Platform 2020, 85).

\section{Die Folgen einer Eskalation: Für Europa steht viel auf dem Spiel}

Die USA bleiben (auch ohne bestehendes Handelsabkommen mit der EU) der wichtigste Handelspartner Deutschlands, wenn die gesamte Leistungsbilanz anstatt allein der Güterhandel betrachtet wird (Braml und Felbermayr, 2021). Jedoch hätte eine Eskalation eines Konflikts mit China negative Auswirkungen für alle Beteiligten. Denn die Wirtschaftsverflechtungen zwischen Deutschland und China sind sehr tiefgreifend. Für den Güterhandel ist China das Hauptimportland sowie das zweitwichtigste Zielland für Deutsche Güterexporte. Vor allem für die Sektoren Elektrotechnik, Textilien, und Elektrische Ausrüstungen spielen die Wertschöpfungsverflechtungen mit China eine bedeutende Rolle (Aichele et al., 2020). Andererseits sind die Lieferkettenabhängigkeiten zwischen den beiden Volkswirtschaften wechselseitig: Deutschland verwendet asiatische Inputs, aber auch China ist abhängig von vielen deutschen Vorprodukten (Aichele et al., 2020). Das bedeutet, dass auch China bei einem Konflikt mit der EU viel zu verlieren hätte.

Eine klare und pragmatische Strategie für die Beziehung mit China notwendig

Die neue EU-Handelsstrategie deutet auf die Bereitschaft der Europäischen Union hin, die Herausforderungen mit China anzugehen und eigene Handelsinteressen selbstbewusst zu vertreten. In der in diesem Frühjahr veröffentlichten Handelsstrategie wird betont, dass „der Aufbau einer faireren und regelbasierten Wirtschaftsbeziehung mit China eine Priorität ist" (European Commission, 2021). Dies ist eine wichtige Ansage der EU an China und die USA, denn seit Jahren kritisieren die USA die starken Marktzugangsbeschränkungen von China. Allerdings ist die EUHandelsstrategie in mehrfacher Hinsicht vage, wie dieses Ziel erreicht werden soll. Außerdem wird die Zusammenarbeit mit den USA nicht reibungslos sein, da einige Konfliktfelder bestehen, wie zum Beispiel die Subventionen der EU im Agrarbereich, die US-Zölle auf Stahl und Aluminium aus der EU oder der Streit um Subventionen für Flugzeughersteller.

Entscheidend ist, dass die EU und die USA eine solide Grundlage für den Umgang mit China ausarbeiten und der Zusammenarbeit an einer WTO-Reform politische Priorität einräumen. Angesichts des steigenden Protektionismus und der zunehmenden Bereitschaft von Ländern, ihre Abhängigkeiten von anderen Staaten zu reduzieren, müssen die USA, China und die EU die Rahmenbedingungen und einen Grundkonsens darüber ausarbeiten, wie sie die globale Wirtschaft voranbringen wollen.

Lisandra Flach

ifo Institut und LMU München flach@ifo.de

\section{Literatur}

Aichele, R., M. Braml und L. Flach (2020), Status quo und Zukunft globaler Lieferketten, ifo Schnelldienst, 73(5), 16-22.

Braml, M. und G. Felbermayr (2021), Die USA sind mit großem Abstand Europas wichtigster Wirtschaftspartner, Kiel Policy Briefs, 150.

Democratic Party Platform (2020), https://democrats.org/where-we-stand/party-platform/.

European Commission (2021), Trade Policy Review - An Open, Sustainable and Assertive Trade Policy, Communication to the European Parliament, European Commission Press Release, 18. Februar.

Flach, L. (2020), Die Handelspolitik mit den USA wird schwierig bleiben, Handelsblatt Online, Homo Oeconomicus, 29. Oktober. 